\title{
On the Multinorm Principle for Finite Abelian Extensions
}

\author{
Timothy P. Pollio
}

\begin{abstract}
Let $L_{1}$ and $L_{2}$ be finite abelian extensions of a global field $K$. We compute the obstruction to the multinorm principle for the pair $L_{1}, L_{2}$.

Keywords: Multinorm, abelian extensions, local-global principle, Tate-Shafarevich group.
\end{abstract}

\section{Introduction}

Let $K$ be a global field. Given a finite extension $L / K$, let $J_{L}$ denote the idele group of $L$, and let $N_{L / K}: J_{L} \rightarrow J_{K}$ denote the natural extension of the norm map associated with $L / K$. When $K$ does not vary, we will write $N\left(L^{\times}\right)$and $N\left(J_{L}\right)$ respectively in place of $N_{L / K}\left(L^{\times}\right)$and $N_{L / K}\left(J_{L}\right)$. A finite extension $L / K$ is said to satisfy the Hasse norm principle if

$$
K^{\times} \cap N\left(J_{L}\right)=N\left(L^{\times}\right) .
$$

The obstruction to the norm principle is given by the Tate-Shafarevich group

$$
\amalg(L / K):=\frac{K^{\times} \cap N\left(J_{L}\right)}{N\left(L^{\times}\right)} .
$$

By analogy, we say that a pair of finite extensions $L_{1}, L_{2}$ of $K$ satisfies the multinorm principle (cf. [7]) if

$$
K^{\times} \cap N\left(J_{L_{1}}\right) N\left(J_{L_{2}}\right)=N\left(L_{1}^{\times}\right) N\left(L_{2}^{\times}\right) .
$$

The obstruction to the multinorm principle is given by the quotient

$$
\amalg\left(L_{1}, L_{2} / K\right):=\frac{K^{\times} \cap N\left(J_{L_{1}}\right) N\left(J_{L_{2}}\right)}{N\left(L_{1}^{\times}\right) N\left(L_{2}^{\times}\right)} .
$$

Received April 17, 2013. 
The multinorm principle has a variety of applications (cf. loc. cit., $\S 1$ ), but it is not fully understood. The main theorem of [7] says that $\amalg\left(L_{1}, L_{2} / K\right)=$ $\{1\}$ whenever $L_{1}, L_{2}$ is a pair of finite separable extensions of $K$ with linearly disjoint Galois closures. However, little is known about $\amalg\left(L_{1}, L_{2} / K\right)$ for more general pairs of extensions.

In this paper, we describe a general approach to the multinorm principle that builds on the techniques used in [7]. The idea is that we should try to describe $\amalg\left(L_{1}, L_{2} / K\right)$ by studying the map

$$
f: \amalg\left(L_{1} / K\right) \times \amalg\left(L_{2} / K\right) \rightarrow \amalg\left(L_{1}, L_{2} / K\right)
$$

defined by

$$
\left(x N\left(L_{1}^{\times}\right), y N\left(L_{2}^{\times}\right)\right) \mapsto x y^{-1} N\left(L_{1}^{\times}\right) N\left(L_{2}^{\times}\right) .
$$

Since $\amalg\left(L_{1}, L_{2} / K\right)$ is determined up to extension by $\operatorname{Im} f$ and Coker $f$, it suffices to describe

$$
\amalg_{1}\left(L_{1}, L_{2} / K\right):=\operatorname{Im}(f) \text { and } \amalg_{2}\left(L_{1}, L_{2} / K\right):=\operatorname{Coker}(f)
$$

individually, and we refer to these groups as the first and second obstructions to the multinorm principle. We will analyze $\amalg_{1}\left(L_{1}, L_{2} / K\right)$ and $\amalg_{2}\left(L_{1}, L_{2} / K\right)$ using group cohomology and class field theory respectively, and prove estimates which will allow us to compute both obstructions in some important special cases. This approach can be used to recover the main theorem of 7] (see §5), and it enables us to prove the main theorem of this note, which characterizes the multinorm principle for pairs of finite abelian extensions.

Theorem 1. Let $L_{1}, L_{2}$ be a pair of finite abelian extensions of $K$. Then

$$
\amalg\left(L_{1}, L_{2} / K\right) \simeq \amalg\left(L_{1} \cap L_{2} / K\right) .
$$

In particular, $L_{1}, L_{2}$ satisfies the multinorm principle iff $L_{1} \cap L_{2}$ satisfies the norm principle.

This result does not extend to non-abelian extensions. In fact, the conclusion of the theorem fails for all of the pairs of extensions constructed in Proposition 12 of [4]. For example, if $K=\mathbb{Q}, L_{1}=\mathbb{Q}(\sqrt{-1}, \sqrt[4]{2})$, and $L_{2}=$ $\mathbb{Q}(\sqrt{-1}, \sqrt{7 \sqrt{2}})$, then $\amalg\left(L_{1} \cap L_{2} / K\right)=\{1\}$, but $\amalg\left(L_{1}, L_{2} / K\right) \simeq \mathbb{Z} / 2 \mathbb{Z}$.

We begin in $\S 2$ and $\S 3$ by giving descriptions of the first and second obstructions respectively. Then we apply these descriptions in the special 
case where $L_{1}, L_{2}$ is a pair of abelian extensions of $K$ to prove Theorem 1 in $\S 4$. Finally, $\S 5$ contains several examples and applications related to our analysis of the multinorm principle.

Remark. Earlier versions of this paper contained additional sufficient conditions for the multinorm principle to hold for $n$-tuples of extensions (see https://sites.google.com/site/timothypollio/papers). By generalizing the proof of Proposition 15 in [7], we were able to show that the multinorm principle holds for $n$-tuples of finite separable extensions whose Galois closures are linearly disjoint as a family and for pairs of Galois extensions with cyclic intersection. Meanwhile, Demarche and Wei obtained similar results which they describe in [4. Their argument is similar to ours and their results are slightly stronger, so we decided to omit these results from the final version of this paper.

If $L_{1}$ and $L_{2}$ are finite abelian extensions of a global field $K$, then they satisfy the hypotheses of Theorem 6 in [4. Let $T$ be the multinorm torus (cf. [7) associated to the pair $L_{1}, L_{2} / K$, and let $S$ be the norm torus associated to $L_{1} \cap L_{2} / K$. Then according to [4], $\amalg_{\omega}^{2}(\widehat{T}) \simeq \amalg_{\omega}^{2}(\widehat{S})$. This does not imply our Theorem 1 since $\amalg_{\omega}^{2}(\widehat{T})$ depends on the defect of weak approximation for $T$ as well as on $\amalg(T)$. However, we can combine this statement with Theorem 1 to get a result for weak approximation. Let $A(T)$ denote the defect of weak approximation for $T$.

Corollary 2. If $L_{1}$ and $L_{2}$ are finite abelian extensions of $K$, and $S$ and $T$ are as above, then

$$
|A(T)|=|A(S)| .
$$

In particular, weak approximation holds for $T$ iff weak approximation holds for $S$.

\section{The First Obstruction}

Fix a separable closure $K_{s}$ of $K$. We will only consider extensions of $K$ which are contained in $K_{s}$. Our analysis of the first obstruction begins with the following observation.

Lemma 3. Let $L_{1}, L_{2}$ be a pair of finite extensions of $K$, let $L=L_{1} L_{2}$, and let

$$
g: \amalg(L / K) \rightarrow \amalg\left(L_{1} / K\right) \times \amalg\left(L_{2} / K\right)
$$


be the map defined by

$$
x N\left(L^{\times}\right) \mapsto\left(x N\left(L_{1}^{\times}\right), x N\left(L_{2}^{\times}\right)\right) .
$$

Then $\amalg_{1}\left(L_{1}, L_{2} / K\right)$ is isomorphic to a quotient of $\operatorname{Coker}(g)$.

Proof. It is clear that $\operatorname{Im}(g) \subset \operatorname{Ker}(f)$, so the claim follows from the first isomorphism theorem.

In this section, we give several results which can be used to compute Coker $(g)$ when $L_{1}$ and $L_{2}$ are Galois extensions of $K$. More generally, if $F$ and $L$ are Galois extensions of $K$ with $L \subset F$, we will describe the map

$$
h: \amalg(F / K)=\frac{K^{\times} \cap N\left(J_{F}\right)}{N\left(F^{\times}\right)} \rightarrow \frac{K^{\times} \cap N\left(J_{L}\right)}{N\left(L^{\times}\right)}=\amalg(L / K)
$$

defined by

$$
x N\left(F^{\times}\right) \mapsto x N\left(L^{\times}\right) .
$$

Given a finite group $G$ and a $G$-module $A$, we let $\hat{H}^{i}(G, A)$ denote the $i$ th Tate cohomology group of $A$, and let Cor, Def, and Rsd denote the corestriction, deflation (cf. [8]), and residuation (cf. [5]) maps. We consider $\mathbb{Z}$ as a $G$-module with trivial action. Let $H_{i}(G, A)$ denote the $i$ th homology group of $A$, and identify $H_{i}(G, A)$ with $\hat{H}^{-i-1}(G, A)$ for $i \geq 1$ (cf. [3, Ch IV, §6]). If $\varphi: G \rightarrow H$ is a group homomorphism, then $\varphi$ induces a map of standard complexes which induces a map of homology groups

$$
\varphi_{*}: H_{i}(G, \mathbb{Z}) \rightarrow H_{i}(H, \mathbb{Z})
$$

(cf. [3, p. 99]). When $A=\mathbb{Z}$, the corestriction and residuation maps can both be interpreted as induced maps in this sense.

Proposition 4. Let $i \geq 1$ and identify $H_{i}(-, \mathbb{Z})$ with $\hat{H}^{-i-1}(-, \mathbb{Z})$.

1) If $G^{\prime}$ is a subgroup of $G$ and $\iota: G^{\prime} \rightarrow G$ is the canonical inclusion map, then

$$
\operatorname{Cor}_{G^{\prime}}^{G}: H_{i}\left(G^{\prime}, \mathbb{Z}\right) \rightarrow H_{i}(G, \mathbb{Z})
$$

is equal to $\iota_{*}$.

2) If $H$ is a quotient of $G$ and $\pi: G \rightarrow H$ is the canonical projection map, then

$$
\operatorname{Rsd}_{H}^{G}: H_{i}(G, \mathbb{Z}) \rightarrow H_{i}(H, \mathbb{Z})
$$

is equal to $\pi_{*}$. 
Proof. This follows from the definitions of the corestriction and residuation maps given in [3, p. 99] and [5].

Let $G=\operatorname{Gal}(F / K)$ and $H=\operatorname{Gal}(L / K)$. For each valuation $v$ of $K$, let $H^{v}$ and $G^{v}$ be the decomposition groups of compatible fixed extensions of $v$ to $L$ and $F$ respectively. Let $\iota_{G}^{v}: G^{v} \rightarrow G$ and $\iota_{H}^{v}: H^{v} \rightarrow H$ denote the canoncial inclusion maps, and let $\pi: G \rightarrow H$ and $\pi^{v}: G^{v} \rightarrow H^{v}$ denote the canonical projection maps. We note that $\iota_{H}^{v} \circ \pi^{v}=\pi \circ \iota_{G}^{v}$, and that the construction of induced maps is functorial so the following is a consequence of Proposition 4.

Corollary 5. For $i \geq 1$ and $A=\mathbb{Z}$,

$$
\operatorname{Cor}_{G^{v}}^{G}=\left(\iota_{G}^{v}\right)_{*}, \quad \operatorname{Cor}_{H^{v}}^{H}=\left(\iota_{H}^{v}\right)_{*}, \quad \operatorname{Rsd}_{H}^{G}=\pi_{*} \quad \text { and } \quad \operatorname{Rsd}_{H^{v}}^{G^{v}}=\pi_{*}^{v} .
$$

Moreover, the diagram

$$
\begin{array}{ccc}
H_{2}\left(G^{v}, \mathbb{Z}\right) & \stackrel{\operatorname{Cor}_{G^{v}}^{G}}{\longrightarrow} & H_{2}(G, \mathbb{Z}) \\
\operatorname{Rsd}_{H^{v}}^{G^{v}} \downarrow & & \operatorname{Rsd}_{H}^{G} \downarrow \\
H_{2}\left(H^{v}, \mathbb{Z}\right) & \stackrel{\operatorname{Cor}_{H^{v}}}{\longrightarrow} & H_{2}(H, \mathbb{Z})
\end{array}
$$

commutes.

Lemma 6. The diagram

$$
\begin{array}{ll}
\bigoplus_{v} H_{2}\left(G^{v}, \mathbb{Z}\right) \stackrel{\sum_{v} \operatorname{Cor}_{G^{v}}^{G}}{\longrightarrow} H_{2}(G, \mathbb{Z}) \\
\left(\operatorname{Rsd}_{H^{v}}^{G^{v}}\right) \downarrow & \operatorname{Rsd}_{H}^{G} \downarrow \\
\bigoplus_{v} H_{2}\left(H^{v}, \mathbb{Z}\right) \stackrel{\sum_{v} \operatorname{Cor}_{H^{v}}^{H}}{\longrightarrow} H_{2}(H, \mathbb{Z})
\end{array}
$$

commutes. Let

$$
\gamma_{F}=\sum_{v} \operatorname{Cor}_{G^{v}}^{G} \quad \text { and } \quad \gamma_{L}=\sum_{v} \operatorname{Cor}_{H^{v}}^{H} .
$$

Then $h$ can be identified with the map

$$
\mathcal{R}_{F / L}: \operatorname{Coker}\left(\gamma_{F}\right) \rightarrow \operatorname{Coker}\left(\gamma_{L}\right)
$$

induced by $\operatorname{Rsd}_{H}^{G}$. 
Corollary 7. $g$ can be identified with the map

$$
\mathcal{R}_{L / L_{1}} \times \mathcal{R}_{L / L_{2}}: \operatorname{Coker}\left(\gamma_{L}\right) \rightarrow \operatorname{Coker}\left(\gamma_{L_{1}}\right) \times \operatorname{Coker}\left(\gamma_{L_{2}}\right) .
$$

Proof of Lemma 6. Both parts of the lemma are proved in [5, §4]. For completeness, we reproduce the argument here. The reader may find it helpful to consult the properties of the deflation and residuation maps described in [7. §3] and to compare the following with the proof of proposition 5 of [7].

Let $C_{L}$ and $C_{F}$ denote the idele class groups of $L$ and $F$ respectively, and identify $H$ with the quotient $G / \operatorname{Gal}(F / L)$. Since the $\operatorname{Gal}(F / L)$-fixed points of

$$
1 \rightarrow F^{\times} \rightarrow J_{F} \rightarrow C_{F} \rightarrow 1
$$

form the short exact sequence

$$
1 \rightarrow L^{\times} \rightarrow J_{L} \rightarrow C_{L} \rightarrow 1,
$$

we have the following commutative diagram with exact rows coming from the long exact sequences in cohomology corresponding to the short exact sequences (2) and (3).

(4)

$$
\begin{aligned}
& \hat{H}^{-1}\left(G, J_{F}\right) \stackrel{\alpha_{F}}{\longrightarrow} \hat{H}^{-1}\left(G, C_{F}\right) \longrightarrow \hat{H}^{0}\left(G, F^{\times}\right) \stackrel{\kappa_{F}}{\longrightarrow} \hat{H}^{0}\left(G, J_{F}\right) \\
& \downarrow \operatorname{Def}_{H}^{G} \quad \downarrow \operatorname{Def}_{H}^{G} \quad \downarrow \operatorname{Def}_{H}^{G} \downarrow \downarrow \operatorname{Def}_{H}^{G} \\
& \hat{H}^{-1}\left(H, J_{L}\right) \stackrel{\alpha_{L}}{\longrightarrow} \hat{H}^{-1}\left(H, C_{L}\right) \longrightarrow \hat{H}^{0}\left(H, L^{\times}\right) \stackrel{\kappa_{L}}{\longrightarrow} \hat{H}^{0}\left(H, J_{L}\right) \text {. }
\end{aligned}
$$

Recall that

$\amalg(L / K)=\frac{K^{\times} \cap N_{L / K}\left(J_{L}\right)}{N_{L / K}\left(L^{\times}\right)}=\operatorname{Ker}\left(\frac{K^{\times}}{N_{L / K}\left(L^{\times}\right)} \rightarrow \frac{J_{K}}{N_{L / K}\left(J_{L}\right)}\right)=\operatorname{Ker}\left(\kappa_{L}\right)$.

Similarly, $\amalg(F / K)=\operatorname{Ker}\left(\kappa_{F}\right)$, so we can identify $h$ with the deflation map

$$
\operatorname{Def}_{H}^{G}: \operatorname{Ker}\left(\kappa_{F}\right) \rightarrow \operatorname{Ker}\left(\kappa_{L}\right) .
$$

Using (4) we identify this with the map

$$
\mathcal{D}: \operatorname{Coker}\left(\alpha_{F}\right) \rightarrow \operatorname{Coker}\left(\alpha_{L}\right)
$$

induced by

$$
\operatorname{Def}_{H}^{G}: \hat{H}^{-1}\left(G, C_{F}\right) \rightarrow \hat{H}^{-1}\left(H, C_{L}\right)
$$


Next, we apply the isomorphisms

$$
\Phi_{G}: \hat{H}^{-1}\left(G, C_{F}\right) \simeq \hat{H}^{-3}(G, \mathbb{Z})=H_{2}(G, \mathbb{Z})
$$

and

$$
\Psi_{G}: \hat{H}^{-1}\left(G, J_{F}\right) \simeq \bigoplus_{v} \hat{H}^{-1}\left(G^{v}, F_{v}^{\times}\right) \simeq \bigoplus_{v} \hat{H}^{-3}\left(G^{v}, \mathbb{Z}\right)=\bigoplus_{v} H_{2}\left(G^{v}, \mathbb{Z}\right)
$$

(cf. [3, Chapter 7]), together with the the corresponding isomorphisms for $H$, to the groups in the left half of (4). The discussion in [3, p. 198] tells us that the diagrams

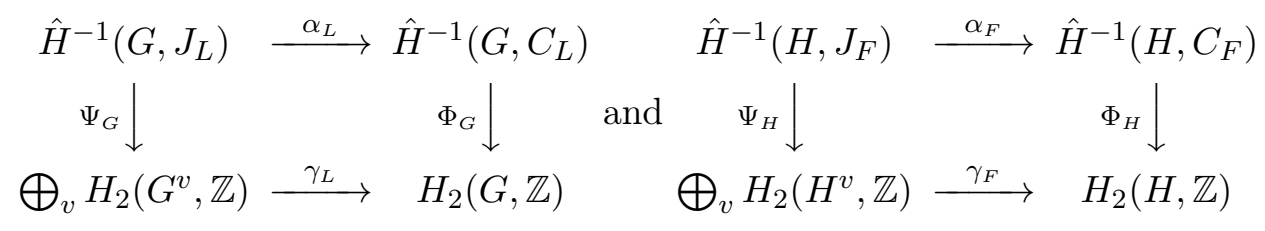

commute, and Theorem 1 in [5] tells us that

$$
\begin{aligned}
& \hat{H}^{-1}\left(G, C_{F}\right) \underset{\Phi_{G}}{\longrightarrow} H_{2}(G, \mathbb{Z}) \\
& \downarrow \operatorname{Def}_{H}^{G} \quad \operatorname{Rsd}_{H}^{G} \downarrow \\
& \hat{H}^{-1}\left(H, C_{L}\right) \stackrel{\Phi_{H}}{\longrightarrow} H_{2}(H, \mathbb{Z})
\end{aligned}
$$

commutes, so these isomorphisms transform

$$
\begin{aligned}
& \hat{H}^{-1}\left(G, J_{F}\right) \stackrel{\alpha_{F}}{\longrightarrow} \hat{H}^{-1}\left(G, C_{F}\right) \quad \bigoplus_{v} H_{2}\left(G^{v}, \mathbb{Z}\right) \stackrel{\gamma_{F}}{\longrightarrow} H_{2}(G, \mathbb{Z}) \\
& \downarrow \operatorname{Def}_{H}^{G} \quad \text { into } \quad \operatorname{Rsd}_{H}^{G} \downarrow \\
& \hat{H}^{-1}\left(H, J_{L}\right) \stackrel{\alpha_{L}}{\longrightarrow} \hat{H}^{-1}\left(H, C_{L}\right) \quad \bigoplus_{v} H_{2}\left(H^{v}, \mathbb{Z}\right) \stackrel{\gamma_{L}}{\longrightarrow} H_{2}(H, \mathbb{Z})
\end{aligned}
$$

Thus, we can identify $\mathcal{D}$ with $\mathcal{R}_{L / F}$. Finally, Corollary 5 tells us that (1) is commutative.

Next we give modified versions of Lemma 6 and Corollary 7 which are more useful for computations by using two different descriptions of the Schur multiplier $H_{2}(-, \mathbb{Z})$. 
Let $G$ be a finite group. Following [2, I.3], we define $M(G)$ to be the group given by the Schur-Hopf formula,

$$
M(G):=\frac{R \cap[F, F]}{[R, F]},
$$

where

$$
1 \rightarrow R \rightarrow F \rightarrow G \rightarrow 1
$$

is any free presentation of $G$. As discussed in loc. cit., the isomorphism class of $M(G)$ is independent of the choice of free presentation, and $M(-)$ becomes a functor from groups to abelian groups once we choose a fixed free presentation for each group $G$.

Lemma 8. For each finite group $G$ there is an isomorphism $H_{2}(G, \mathbb{Z}) \simeq$ $M(G)$. These isomorphisms can be chosen such that for all homomorphisms of finite groups $\varphi: G \rightarrow H$, the natural diagram

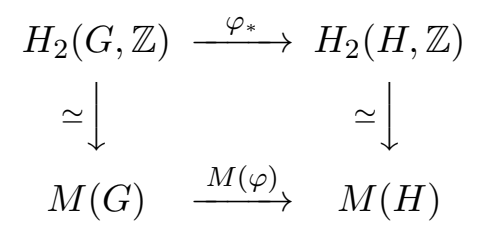

commutes.

Proof. This follows from Proposition 5.5 of [2, p. 51].

Lemma 9. The diagram

$$
\begin{array}{cc}
\bigoplus_{v} M\left(G^{v}\right) \stackrel{\sum_{v} M\left(\iota_{G}^{v}\right)}{\longrightarrow} M(G) \\
\left(M\left(\pi^{v}\right)\right) \downarrow \\
\bigoplus_{v} M\left(H^{v}\right) \stackrel{\sum_{v} M\left(\iota_{H}^{v}\right)}{\longrightarrow} M(H)
\end{array}
$$

commutes. Let

$$
\delta_{F}:=\sum_{v} M\left(\iota_{G}^{v}\right) \quad \text { and } \quad \delta_{L}:=\sum_{v} M\left(\iota_{H}^{v}\right) .
$$

Then $h$ can be identified with the map

$$
\mathcal{S}_{F / L}: \operatorname{Coker}\left(\delta_{F}\right) \rightarrow \operatorname{Coker}\left(\delta_{L}\right)
$$

induced by $M(\pi)$. 
Proof. We obtain (5) by applying Lemmas 4 and 8 to (1). This proves the commutivity of (5) and allows us to identify $\mathcal{R}_{F / L}$ with $\mathcal{S}_{F / L}$.

Corollary 10. $g$ can be identified with the map

$$
\mathcal{S}_{L / L_{1}} \times \mathcal{S}_{L / L_{2}}: \operatorname{Coker}\left(\delta_{L}\right) \rightarrow \operatorname{Coker}\left(\delta_{L_{1}}\right) \times \operatorname{Coker}\left(\delta_{L_{2}}\right) .
$$

The exterior square of a finite abelian group $G$ is defined as

$$
G \wedge G:=G \otimes G /\langle g \otimes g \mid g \in G\rangle .
$$

Lemma 11. If $G$ is abelian, then $M(G)$ is naturally isomorphic to $G \wedge G$. That is, for every homomorphism of finite abelian groups $\varphi: G \rightarrow H$ there is a commutative diagram of the form

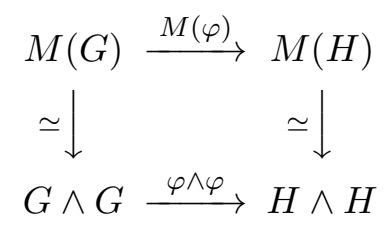

where $\varphi \wedge \varphi$ is the map induced by $\varphi \otimes \varphi$.

Proof. This follows from 4.5 and 4.7 in [2, I.4].

Lemma 12. The diagram

$$
\begin{aligned}
\bigoplus_{v} G^{v} \wedge G^{v} \stackrel{\sum_{v} \iota_{G}^{v} \wedge \iota_{G}^{v}}{\longrightarrow} G \wedge G \\
\left(\pi^{v} \wedge \pi^{v}\right) \downarrow \\
\bigoplus_{v} H^{v} \wedge H^{v} \stackrel{\sum_{v} \iota_{H}^{v} \wedge \iota_{H}^{v}}{\longrightarrow} H \wedge H
\end{aligned}
$$

commutes. Let

$$
\epsilon_{F}:=\sum_{v} \iota_{G}^{v} \wedge \iota_{G}^{v} \quad \text { and } \quad \epsilon_{L}:=\sum_{v} \iota_{H}^{v} \wedge \iota_{H}^{v} .
$$

Then $h$ can be identified with the map

$$
\mathcal{T}_{F / L}: \operatorname{Coker}\left(\epsilon_{F}\right) \rightarrow \operatorname{Coker}\left(\epsilon_{L}\right)
$$

induced by $\pi \wedge \pi$. 
Proof. We obtain (6) by applying Lemma 11 to (5). This proves the commutivity of (6) and allows us to identify $\mathcal{S}_{F / L}$ with $\mathcal{T}_{F / L}$.

Corollary 13. $g$ can be identified with the map

$$
\mathcal{T}_{L / L_{1}} \times \mathcal{T}_{L / L_{2}}: \operatorname{Coker}\left(\epsilon_{L}\right) \rightarrow \operatorname{Coker}\left(\epsilon_{L_{1}}\right) \times \operatorname{Coker}\left(\epsilon_{L_{2}}\right) .
$$

\section{The Second Obstruction}

We begin this section by constructing an exact sequence that contains the map

$$
f: \amalg\left(L_{1} / K\right) \times \amalg\left(L_{2} / K\right) \rightarrow \amalg\left(L_{1}, L_{2} / K\right)
$$

defined by

$$
\left(x N\left(L_{1}^{\times}\right), y N\left(L_{2}^{\times}\right)\right) \mapsto x y^{-1} N\left(L_{1}^{\times}\right) N\left(L_{2}^{\times}\right) .
$$

Proposition 14. If $L_{1}, L_{2}$ is a pair of finite extensions of $K$, then there is an exact sequence of the form

$$
\begin{gathered}
1 \rightarrow \frac{K^{\times} \cap N\left(J_{L_{1}}\right) \cap N\left(J_{L_{2}}\right)}{N\left(L_{1}^{\times}\right) \cap N\left(L_{2}^{\times}\right)} \rightarrow \amalg\left(L_{1} / K\right) \times \amalg\left(L_{2} / K\right) \stackrel{f}{\rightarrow} \\
\amalg\left(L_{1}, L_{2} / K\right) \rightarrow \frac{J_{K}}{K^{\times}\left(N\left(J_{L_{1}}\right) \cap N\left(J_{L_{2}}\right)\right)} \rightarrow \\
\frac{J_{K}}{K^{\times} N\left(J_{L_{1}}\right)} \times \frac{J_{K}}{K^{\times} N\left(J_{L_{2}}\right)} \rightarrow \frac{J_{K}}{K^{\times} N\left(J_{L_{1}}\right) N\left(J_{L_{2}}\right)} \rightarrow 1 .
\end{gathered}
$$

We can think of this as a generalization of Proposition 1 in [7]. The proof of Proposition 14 uses the following elementary lemma.

Lemma 15. Let $\mathcal{A}$ be an abelian group with subgroups $\mathcal{B}$ and $\mathcal{C}$. The sequence

$$
1 \longrightarrow \frac{\mathcal{A}}{\mathcal{A} \cap \mathcal{B}} \stackrel{\varphi}{\longrightarrow} \frac{\mathcal{A}}{\mathcal{B}} \times \frac{\mathcal{A}}{\mathcal{C}} \stackrel{\psi}{\longrightarrow} \frac{\mathcal{A}}{\mathcal{B C}} \longrightarrow 1,
$$

where $\varphi$ and $\psi$ are defined by

$$
\varphi(x \mathcal{A} \cap \mathcal{B})=(x \mathcal{B}, x \mathcal{C}) \text { and } \psi(x \mathcal{B}, y \mathcal{C})=x y^{-1} \mathcal{B C},
$$

is exact. 
Proof of Proposition 14. We obtain the commutative diagram

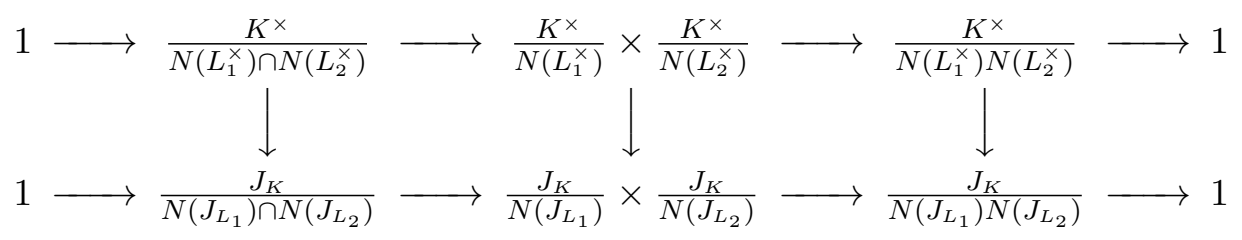

by applying Lemma 15 twice. The top row corresponds to $\mathcal{A}=K^{\times}, \mathcal{B}=$ $N\left(L_{1}^{\times}\right)$, and $\mathcal{C}=N\left(L_{2}^{\times}\right)$, while the bottom row corresponds to $\mathcal{A}=J_{K}, \mathcal{B}=$ $N\left(J_{L_{1}}\right)$, and $\mathcal{C}=N\left(J_{L_{2}}\right)$. The vertical maps are induced by the inclusion $K^{\times} \rightarrow J_{K}$. Applying the snake lemma to this diagram gives (7).

It follows from the exactness of (7) that

$$
\amalg_{2}\left(L_{1}, L_{2} / K\right) \simeq \frac{K^{\times} N\left(J_{L_{1}}\right) \cap K^{\times} N\left(J_{L_{2}}\right)}{K^{\times}\left(N\left(J_{L_{1}}\right) \cap N\left(J_{L_{2}}\right)\right)} .
$$

We give an upper bound for the order of $\amalg_{2}\left(L_{1}, L_{2} / K\right)$ by using class field theory to estimate the order of this quotient. For a global field $F$, we let $C_{F}$ denote the idele class group of $F$.

Lemma 16. Let $L_{1}, L_{2}$ be a pair of finite extensions of $K$, and let $L=L_{1} L_{2}$. If $M_{i}$ is the maximal abelian subextension of $L_{i} / K$, and $M$ is the maximal abelian subextension of $L / K$, then

$$
\left|\amalg_{2}\left(L_{1}, L_{2} / K\right)\right| \leq \frac{[M: K]}{\left[M_{1} M_{2}: K\right]} .
$$

In particular, if $M=M_{1} M_{2}$, then the second obstruction is trivial.

Proof. Clearly $N\left(J_{L}\right) \leq N\left(J_{L_{1}}\right) \cap N\left(J_{L_{2}}\right)$, so it follows from (8) that $\amalg_{2}\left(L_{1}, L_{2} / K\right)$ is isomorphic to a quotient of

$$
\frac{K^{\times} N\left(J_{L_{1}}\right) \cap K^{\times} N\left(J_{L_{2}}\right)}{K^{\times} N\left(J_{L}\right)} \simeq \frac{N\left(C_{L_{1}}\right) \cap N\left(C_{L_{2}}\right)}{N\left(C_{L}\right)} .
$$

According to [3, Exercise 8],

$$
N\left(C_{L}\right)=N\left(C_{M}\right), \quad N\left(C_{L_{i}}\right)=N\left(C_{M_{i}}\right),
$$

by [1, p. 55],

$$
N\left(C_{M_{1}}\right) \cap N\left(C_{M_{2}}\right)=N\left(C_{M_{1} M_{2}}\right)
$$


and by [3, p. 172 Theorem $5.1 \mathrm{~B}]$,

$$
\left[C_{K}: N\left(C_{M}\right)\right]=[M: K] \quad \text { and } \quad\left[C_{K}: N\left(C_{M_{1} M_{2}}\right)\right]=\left[M_{1} M_{2}: K\right],
$$

so

$$
\left|\amalg_{2}\left(L_{1}, L_{2} / K\right)\right| \leq\left|\frac{N\left(C_{L_{1}}\right) \cap N\left(C_{L_{2}}\right)}{N\left(C_{L}\right)}\right|=\left|\frac{N\left(C_{M_{1} M_{2}}\right)}{N\left(C_{M}\right)}\right|=\frac{[M: K]}{\left[M_{1} M_{2}: K\right]} .
$$

Another approach to the second obstruction is to consider the map

$$
\varphi: J_{L_{1}} / L_{1}^{\times} N_{L / L_{1}}\left(J_{L}\right) \times J_{L_{2}} / L_{2}^{\times} N_{L / L_{2}}\left(J_{L}\right) \longrightarrow J_{K} / K^{\times} N_{L / K}\left(J_{L}\right)
$$

induced by the product of norm maps $N_{L_{1} / K}$ and $N_{L_{2} / K}$ as in [6] and [7].

Lemma 17. If $\varphi$ is injective, then

$$
K^{\times} \cap N\left(J_{L_{1}}\right) N\left(J_{L_{2}}\right)=\left(K^{\times} \cap N\left(J_{L}\right)\right) N\left(L_{1}^{\times}\right) N\left(L_{2}^{\times}\right) .
$$

In particular, $\amalg_{2}\left(L_{1}, L_{2} / K\right)=\{1\}$.

Proof. See the proof of Lemma 3 in [7].

Corollary 18. If $L_{1} \subset L_{2}$ and $\varphi$ is injective, then the natural map

$$
\amalg\left(L_{2} / K\right) \rightarrow \amalg\left(L_{1} / K\right)
$$

is surjective.

Proof. In this case, 10 takes the form

$$
K^{\times} \cap N\left(J_{L_{1}}\right)=\left(K^{\times} \cap N\left(J_{L_{2}}\right)\right) N\left(L_{1}^{\times}\right) .
$$

Lemma 3 of [7] says that $\varphi$ is a bijection if $L_{1}, L_{2}$ is a pair of linearly disjoint Galois extensions of $K$. This result can be strengthened as follows.

Lemma 19. Let $L_{1}, L_{2}$ be a pair of Galois extensions of $K$, let $L=L_{1} L_{2}$ and $E=L_{1} \cap L_{2}$, and let $(-)^{a b}$ denote the abelianization functor. $\varphi$ is injective iff the natural map $\operatorname{Gal}(L / E)^{a b} \rightarrow \operatorname{Gal}(L / K)^{a b}$ is injective. In particular, $\varphi$ is injective whenever $L_{1}$ and $L_{2}$ are both abelian extensions of $K$. 
Proof. We can factor $\varphi$ as

$$
\begin{gathered}
J_{L_{1}} / L_{1}^{\times} N_{L / L_{1}}\left(J_{L}\right) \times J_{L_{2}} / L_{2}^{\times} N_{L / L_{2}}\left(J_{L}\right) \stackrel{\varphi_{0}}{\longrightarrow} J_{E} / E^{\times} N_{L / E}\left(J_{L}\right) \stackrel{N_{E / K}}{\longrightarrow} \\
J_{K} / K^{\times} N_{L / K}\left(J_{L}\right),
\end{gathered}
$$

where $\varphi_{0}$ is induced by the product of the norm maps $N_{L_{1} / E}$ and $N_{L_{2} / E}$. $L_{1}, L_{2}$ is a pair of linearly disjoint Galois extensions of $E$, so $\varphi_{0}$ is an isomorphism. It follows that $\varphi$ is injective iff $N_{E / K}$ is injective. Since the Tate isomorphisms commute with corestriction [3, p. 197], there is a commutative diagram

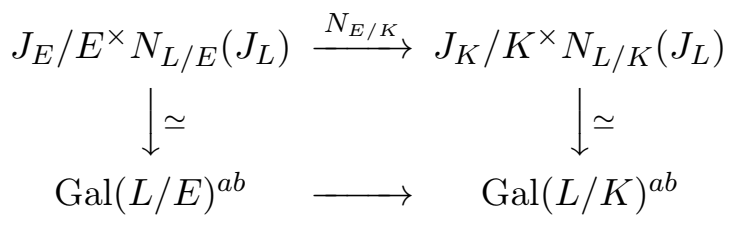

and the claim follows.

\section{Proof of Theorem 1}

Let $L_{1}, L_{2}$ be a pair of finite abelian extensions of $K$, let $L=L_{1} L_{2}$, and let $E=L_{1} \cap L_{2}$. We begin the proof of Theorem 1 by computing Coker $(g)$.

Lemma 20.

$$
\operatorname{Coker}(g) \simeq \amalg(E / K) .
$$

Let $G, G_{i}$, and $G_{E}$ denote the Galois groups $\operatorname{Gal}(L / K), \operatorname{Gal}\left(L_{i} / K\right)$, and $\operatorname{Gal}(E / K)$ respectively, and let $G^{v}, G_{i}^{v}$, and $G_{E}^{v}$ denote the decomposition groups of (compatible) fixed extensions of $v$ to the fields $L, L_{i}$, and $E$. Let $\pi_{i}: G \rightarrow G_{i}, \rho: G \rightarrow G_{E}, \rho_{i}: G_{i} \rightarrow G_{E}$, and $\rho_{i}^{v}: G_{i}^{v} \rightarrow G_{E}^{v}$ denote the canonical projection maps, and let $\iota^{v}: G^{v} \rightarrow G, \iota_{i}^{v}: G_{i}^{v} \rightarrow G_{i}$, and $\iota_{E}^{v}: G_{E}^{v} \rightarrow$ $G_{E}$ denote the canonical inclusion maps.

By Lemma 12 and Corollary 13 there are commutative diagrams

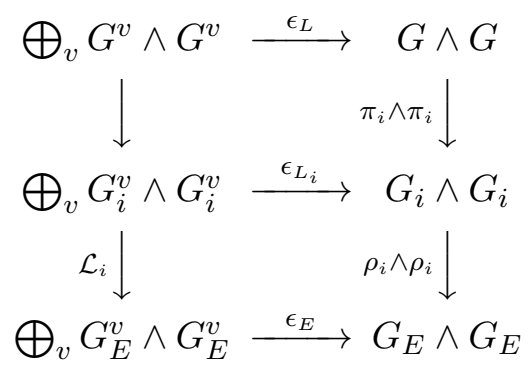


for $i=1,2$ where

$$
\begin{gathered}
\epsilon_{L}=\sum_{v} \iota^{v} \wedge \iota^{v}, \quad \epsilon_{L_{i}}=\sum_{v} \iota_{i}^{v} \wedge \iota_{i}^{v}, \\
\epsilon_{E}=\sum_{v} \iota_{E}^{v} \wedge \iota_{E}^{v}, \quad \text { and } \quad \mathcal{L}_{i}=\bigoplus_{v}\left(\rho_{i}^{v} \wedge \rho_{i}^{v}\right),
\end{gathered}
$$

and we can identify $g$ with the map

$$
\mathcal{T}: \operatorname{Coker}\left(\epsilon_{L}\right) \rightarrow \operatorname{Coker}\left(\epsilon_{L_{1}}\right) \times \operatorname{Coker}\left(\epsilon_{L_{2}}\right)
$$

induced by the map

$$
\mathcal{T}_{0}: G \wedge G \rightarrow\left(G_{1} \wedge G_{1}\right) \times\left(G_{2} \wedge G_{2}\right)
$$

defined by

$$
\mathcal{T}_{0}(a \wedge b)=\left(\pi_{1}(a) \wedge \pi_{1}(b), \pi_{2}(a) \wedge \pi_{2}(b)\right)
$$

for $a, b \in G$.

We start by analyzing $\mathcal{T}_{0}$. Given subsets $A$ and $B$ of an abelian group $C$, we let $A \wedge B$ denote the set of all sums in $C \wedge C$ of the form $\sum_{i} a_{i} \wedge b_{i}$ with $a_{i} \in A$ and $b_{i} \in B$. Let $\mu$ be a fixed section of $\rho$ and define a section $\mu_{1}$ of $\rho_{1}$ by $\mu_{1}=\pi_{1} \circ \mu$.

\section{Lemma 21.}

$$
\begin{aligned}
& \left(\mu_{1}\left(G_{E}\right) \wedge \operatorname{Ker}\left(\rho_{1}\right), 0\right)=\mathcal{T}_{0}\left(\mu\left(G_{E}\right) \wedge \operatorname{Gal}\left(L / L_{2}\right)\right), \\
& \left(\operatorname{Ker}\left(\rho_{1}\right) \wedge \mu_{1}\left(G_{E}\right), 0\right)=\mathcal{T}_{0}\left(\operatorname{Gal}\left(L / L_{2}\right) \wedge \mu\left(G_{E}\right)\right),
\end{aligned}
$$

and

$$
\left(\operatorname{Ker}\left(\rho_{1}\right) \wedge \operatorname{Ker}\left(\rho_{1}\right), 0\right)=\mathcal{T}_{0}\left(\operatorname{Gal}\left(L / L_{2}\right) \wedge \operatorname{Gal}\left(L / L_{2}\right)\right)
$$

Proof. Direct computation. We note that $\pi_{1}\left(\operatorname{Gal}\left(L / L_{2}\right)\right)=\operatorname{Gal}\left(L_{1} / E\right)=$ $\operatorname{Ker}\left(\rho_{1}\right), \pi_{2}\left(\operatorname{Gal}\left(L / L_{2}\right)\right)=1, \pi_{1}\left(\mu\left(G_{E}\right)\right)=\mu_{1}\left(G_{E}\right)$, and that $g_{1} \wedge g_{2}=0$ whenever $g_{2}=1$.

These identities allow us to compute the cokernel of $\mathcal{T}_{0}$. 


\section{Lemma 22.}

$$
\operatorname{Coker}\left(\mathcal{T}_{0}\right) \simeq G_{E} \wedge G_{E}
$$

Proof. Since Coker $\left(\mathcal{T}_{0}\right)$ and $G_{E} \wedge G_{E}$ are finite groups, it suffices to construct a surjective homomorphism from each to the other. We define a surjective homomorphism

$$
P_{0}:\left(G_{1} \wedge G_{1}\right) \times\left(G_{2} \wedge G_{2}\right) \rightarrow G_{E} \wedge G_{E}
$$

by

$$
P_{0}(a \wedge b, 0)=\rho_{1}(a) \wedge \rho_{1}(b) \quad \text { and } \quad P_{0}(0, c \wedge d)=-\rho_{2}(c) \wedge \rho_{2}(d)
$$

for $a, b \in G_{1}$ and $c, d \in G_{2} . P_{0} \circ \mathcal{T}_{0}=0$, so $P_{0}$ induces a surjective homomorphism

$$
P: \operatorname{Coker}\left(\mathcal{T}_{0}\right) \rightarrow G_{E} \wedge G_{E}
$$

To get a homomorphism in the other direction, we first define a set map

$$
S_{0}: G_{E} \times G_{E} \rightarrow \operatorname{Coker}\left(\mathcal{T}_{0}\right)
$$

by

$$
S_{0}(e, f)=\left(\mu_{1}(e) \wedge \mu_{1}(f), 0\right)+\operatorname{Im}\left(\mathcal{T}_{0}\right)
$$

for $e, f \in G_{E}$. If $e_{1}, e_{2} \in G_{E}$, then $\mu_{1}\left(e_{1} e_{2}\right) \mu_{1}\left(e_{1}\right)^{-1} \mu_{1}\left(e_{2}\right)^{-1} \in \operatorname{Ker}\left(\rho_{1}\right)$, so it follows from Lemma 21 that

$$
\begin{gathered}
S_{0}\left(e_{1} e_{2}, f\right)-S_{0}\left(e_{1}, f\right)-S_{0}\left(e_{2}, f\right)= \\
\left(\mu_{1}\left(e_{1} e_{2}\right) \mu_{1}\left(e_{1}\right)^{-1} \mu_{1}\left(e_{2}\right)^{-1} \wedge \mu_{1}(f), 0\right)+\operatorname{Im}\left(\mathcal{T}_{0}\right)=0 .
\end{gathered}
$$

A similar calculation can be done for the second argument, so $S_{0}$ is bilinear and induces a homomorphism $S: G_{E} \wedge G_{E} \rightarrow \operatorname{Coker}\left(\mathcal{T}_{0}\right)$. It remains to show 
that $S$ is surjective. Since $\pi_{2} \wedge \pi_{2}$ is surjective, we have

$$
\operatorname{Coker}\left(\mathcal{T}_{0}\right)=\left(G_{1} \wedge G_{1}, 0\right)+\operatorname{Im}\left(\mathcal{T}_{0}\right)
$$

and it suffices to show that

$$
\left(g_{1} \wedge g_{2}, 0\right)+\operatorname{Im}\left(\mathcal{T}_{0}\right)=\left(\left(\mu_{1} \circ \rho_{1}\right)\left(g_{1}\right) \wedge\left(\mu_{1} \circ \rho_{1}\right)\left(g_{2}\right), 0\right)+\operatorname{Im}\left(\mathcal{T}_{0}\right)
$$

for all $g_{1}, g_{2} \in G_{1}$. Since

$$
\left(\mu_{1} \circ \rho_{1}\right)\left(g_{i}\right) g_{i}^{-1} \in \operatorname{Ker}\left(\rho_{1}\right),
$$

for $i=1,2$, this follows from Lemma 21 .

Proof of Lemma 20. Since $g$ can be identified with $\mathcal{T}$ and $\amalg(E / K)$ can be identified with $\operatorname{Coker}\left(\epsilon_{E}\right)$, it suffices to prove that $\operatorname{Coker}(\mathcal{T}) \simeq \operatorname{Coker}\left(\epsilon_{E}\right) . P$ induces a homomorphism

$$
\mathcal{P}: \operatorname{Coker}(\mathcal{T}) \rightarrow \operatorname{Coker}\left(\epsilon_{E}\right)
$$

which must be surjective since $P$ is surjective. Since the maps $\mathcal{L}_{i}$ in 11 are surjective, and since $P$ is injective, a short diagram chase shows that $\mathcal{P}$ must also be injective.

Proof of Theorem 1. By Lemma 16, $\amalg_{2}\left(L_{1}, L_{2} / K\right)=\{1\}$, so $\amalg\left(L_{1}, L_{2} / K\right)=\amalg_{1}\left(L_{1}, L_{2} / K\right)$. According to Lemmas 3 and 20 . $\amalg\left(L_{1}, L_{2} / K\right)$ is isomorphic to a quotient of $\amalg(E / K)$. Since both of these groups are finite, it suffices to show that $\amalg(E / K)$ is isomorphic to a quotient of $\amalg\left(L_{1}, L_{2} / K\right)$. Consider the map

$$
j: \amalg(L / K) \rightarrow \amalg(L, E / K)=\amalg(E / K)
$$

defined by

$$
x N\left(L^{\times}\right) \mapsto x N\left(E^{\times}\right) .
$$

$L, E$ is a pair of abelian extensions of $K$, so Lemma 19 and Corollary 18 guarantee that $j$ is surjective. $j$ factors through $\amalg\left(L_{1}, L_{2} / K\right)$, so $\amalg(E / K)$ is a homomorphic image of $\amalg\left(L_{1}, L_{2} / K\right)$. 


\section{Examples and Discussion}

In this section we describe several applications of the methods developed in the previous sections and discuss some problems related to the multinorm principle.

Example 1. If $L_{1}, L_{2}$ is a linearly disjoint pair of finite Galois extensions of a global field $K$, then we can recover the main theorem of [7] by using the results from $\S 2$ and $\S 3$ to prove that $\amalg\left(L_{1}, L_{2} / K\right)=\{1\}$. To prove that $\amalg_{1}\left(L_{1}, L_{2} / K\right)=\{1\}$, it suffices to show that

$$
g: \amalg(L / K) \rightarrow \amalg\left(L_{1} / K\right) \times \amalg\left(L_{2} / K\right)
$$

is surjective. Let $G=\operatorname{Gal}(L / K), G_{i}=\operatorname{Gal}\left(L_{i} / K\right)$, and let $\pi_{i}: G \rightarrow G_{i}$ be the natural projection map. By Corollary 10 it suffices to show that

$$
M\left(\pi_{1}\right) \times M\left(\pi_{2}\right): M(G) \rightarrow M\left(G_{1}\right) \times M\left(G_{2}\right)
$$

is surjective. Let $\iota_{i}: G_{i} \rightarrow G$ be the monomorphism corresponding to the natural identification

$$
G_{i}=\operatorname{Gal}\left(L_{i} / K\right) \simeq \operatorname{Gal}\left(L / L_{3-i}\right) \leq G .
$$

Then $\pi_{i} \circ \iota_{i}=\operatorname{id}_{G_{i}}$ and $\pi_{3-i} \circ \iota_{i}$ is the trivial homomorphism, so $M\left(\pi_{i}\right) \circ$ $M\left(\iota_{i}\right)=\operatorname{id}_{M\left(G_{i}\right)}$ while $M\left(\pi_{3-i}\right) \circ M\left(\iota_{i}\right)$ is the zero map, and the surjectivity of (12) follows.

If $M_{i}$ and $M$ denote the maximal abelian subextensions of $L_{i} / K$ and $L_{1} L_{2} / K$ respectively, then $M=M_{1} M_{2}$, so $\amalg_{2}\left(L_{1}, L_{2} / K\right)=\{1\}$ by Lemma 16.

Example 2. In light of Theorem 1, it is natural to ask what can be said about the multinorm principle for $n$-tuples of abelian extensions. Unfortunately, the conclusion of Theorem 1 can fail for $n \geq 3$. That is, there are $n$-tuples of abelian extensions which do not satisfy the multinorm principle even though their intersection satisfies the norm principle. For example, if $K=\mathbb{Q}, L_{1}=\mathbb{Q}(\sqrt{13}), L_{2}=\mathbb{Q}(\sqrt{17})$, and $L_{3}=\mathbb{Q}(\sqrt{13 \cdot 17})$ are the extensions from Example 2 in [7], then

$$
\amalg\left(L_{1}, L_{2}, L_{3} / K\right):=\frac{K^{\times} \cap N\left(J_{L_{1}}\right) N\left(J_{L_{2}}\right) N\left(J_{L_{3}}\right)}{N\left(L_{1}^{\times}\right) N\left(L_{2}^{\times}\right) N\left(L_{3}^{\times}\right)} \simeq \mathbb{Z} / 2 \mathbb{Z}
$$


even though $L_{1} \cap L_{2} \cap L_{3}=K$. (So $L_{1} \cap L_{2} \cap L_{3} / K$ trivially satisfies the norm principle.)

One of the key ingredients in our proof of Theorem 1 is the fact that the map

$$
f: \amalg\left(L_{1} / K\right) \times \amalg\left(L_{2} / K\right) \rightarrow \amalg\left(L_{1}, L_{2} / K\right)
$$

defined in $\S 1$ is surjective for every pair of abelian extensions. We can define analogous maps for $n \geq 3$, but we cannot get surjectivity for all families of extensions. For example, the map

$$
\amalg\left(L_{1} / K\right) \times \amalg\left(L_{2} / K\right) \times \amalg\left(L_{3} / K\right) \rightarrow \amalg\left(L_{1}, L_{2}, L_{3} / K\right)
$$

defined by

$$
\left(x N\left(L_{1}^{\times}\right), y N\left(L_{2}^{\times}\right), z N\left(L_{3}^{\times}\right)\right) \mapsto x y z N\left(L_{1}^{\times}\right) N\left(L_{2}^{\times}\right) N\left(L_{3}^{\times}\right)
$$

is not surjective for the extensions in Example 2 since each individual extension satisfies the norm principle and all of the groups $\amalg\left(L_{i} / K\right)$ are trivial. It does not help to add other Tate-Shafarevich groups to the domain. For example, the natural map $\amalg\left(L_{1} L_{2} / K\right) \rightarrow \amalg\left(L_{1}, L_{2}, L_{3} / K\right)$ factors through $\amalg\left(L_{1} / K\right)$, so incorporating it into the definition above does not enlarge the image.

Another generalization of $f$ is the map

$$
f_{3}: \amalg\left(L_{1} / K\right) \times \amalg\left(L_{2}, L_{3} / K\right) \rightarrow \amalg\left(L_{1}, L_{2}, L_{3} / K\right)
$$

defined by

$$
\left(x N\left(L_{1}^{\times}\right), y N\left(L_{2}^{\times}\right) N\left(L_{3}^{\times}\right)\right) \mapsto x y^{-1} N\left(L_{1}^{\times}\right) N\left(L_{2}^{\times}\right) N\left(L_{3}^{\times}\right) .
$$

We can construct an exact sequence analogous to (7) that contains $f_{3}$ by adapting the proof of Proposition 14, but this is rarely sufficient to compute $\amalg\left(L_{1}, L_{2}, L_{3} / K\right)$. The domain is trivial for the extensions of Example 2, so $f_{3}$ can also fail to be surjective.

Example 3. If $L_{1}, L_{2}$ is a pair of finite extensions of a global field $K$, let us say that $L_{1}, L_{2}$ satisfies the intersection principle if

$$
K^{\times} \cap N\left(J_{L_{1}}\right) \cap N\left(J_{L_{2}}\right)=N\left(L_{1}^{\times}\right) \cap N\left(L^{\times}\right) .
$$


The obstruction to this local-global principle is given by

$$
\amalg_{\cap}\left(L_{1}, L_{2} / K\right):=\frac{K^{\times} \cap N\left(J_{L_{1}}\right) \cap N\left(J_{L_{2}}\right)}{N\left(L_{1}^{\times}\right) \cap N\left(L^{\times}\right)} .
$$

This group naturally arises as the first nontrivial term in (7), and we can truncate (7) to obtain the short exact sequence

$$
1 \rightarrow \amalg_{\cap}\left(L_{1}, L_{2} / K\right) \rightarrow \amalg\left(L_{1} / K\right) \times \amalg\left(L_{2} / K\right) \rightarrow \amalg_{1}\left(L_{1}, L_{2} / K\right) \rightarrow 1 .
$$

One possibility this suggests is that we may be able to learn about the first obstruction indirectly by studying the intersection problem. On the other hand, we can use (13) to determine if the intersection principle holds whenever we understand $\amalg_{1}\left(L_{1}, L_{2} / K\right)$. In particular, we have the following corollaries to the main theorem of [7] and Theorem 1 of this paper.

Corollary 23. If $L_{1}, L_{2}$ is a pair of finite separable extensions of $K$ with linearly disjoint Galois closures, then

$$
\amalg_{\cap}\left(L_{1}, L_{2} / K\right) \simeq \amalg\left(L_{1} / K\right) \times \amalg\left(L_{2} / K\right) .
$$

Corollary 24. If $L_{1}, L_{2}$ is a pair of finite abelian extensions of $K$, then

$$
\left|\amalg_{\cap}\left(L_{1}, L_{2} / K\right)\right|=\frac{\left|\amalg\left(L_{1} / K\right)\right|\left|\amalg\left(L_{2} / K\right)\right|}{\left|\amalg\left(L_{1} \cap L_{2} / K\right)\right|} .
$$

Example 4. The map $\varphi$ defined in $\S 3$ may fail to be injective even if $E=L_{1} \cap L_{2}$ is a cyclic extension of $K$. Let $K=\mathbb{Q}, L_{1}=\mathbb{Q}\left(i, 2^{1 / 4}\right)$, and $L_{2}=$ $\mathbb{Q}(\sqrt{2}, \sqrt{3})$. Then $L=\mathbb{Q}\left(i, 2^{1 / 4}, \sqrt{3}\right)$ and $E=\mathbb{Q}(\sqrt{2})$. Let $G=\operatorname{Gal}(L / K)$, let $H=\operatorname{Gal}(L / E)$, let $\tau \in H$ be the automorphism defined by complex conjugation, and let $\sigma \in G$ be the automorphism which sends $2^{1 / 4}$ to $i 2^{1 / 4}$ and fixes $i$ and $\sqrt{3}$. Then $H=H^{a b}$ and $[\sigma, \tau]$ is a non-trivial element of $\operatorname{Ker}\left(H^{a b} \rightarrow G^{a b}\right)$, so it follows from Lemma 19 that $\varphi$ is not injective.

\section{References}

1. E. Artin, J. Tate, Class Field Theory, AMS Chelsea, 2009.

2. F. R. Beyl, J. Tappe, Group Extensions, Representations, and the Schur Multiplicator, Springer-Verlag, 1982. 
3. J.W.S. Cassels, A. Frölich (Eds.), Algebraic Number Theory, Thompson Book Company Inc., Washington D.C., 1967.

4. C. Demarche, D. Wei, Hasse Principle and Weak Approximation for Multinorm Equations, ArXiv:1212.5889.

5. K. Horie, M. Horie, Deflation and residuation for class formation, Journal of Algebra 245(2001), 607-619.

6. V.P. Platonov, A.S. Rapinchuk, Algebraic Groups and Number Theory, Academic Press, 1994.

7. T. Pollio, A.S. Rapinchuk, The multinorm principle for linearly disjoint Galois extensions, Journal of Number Theory 133(2013), 802-821.

8. E. Weiss, A deflation map, J. Math. Mech. 8(1959), 309-329.

Timothy Pollio

Department of Mathematics

Kerchof Hall, P.O. Box 400137

University of Virginia

Charlottesville, VA 22904

E-mail: timothypollio@gmail.com 